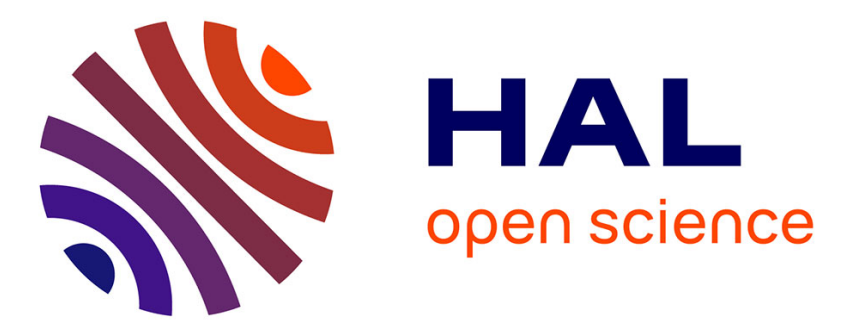

\title{
Epitaxial strain and thickness dependent structural, electrical and magnetic properties of La0.67Sr0.33MnO3 films
}

\author{
Sandeep Kumar Chaluvadi, Fernando Ajejas, Pasquale Orgiani, Sylvain \\ Lebargy, Albert Minj, Stéphane Flament, Julio Camarero, Paolo Perna, \\ Laurence Méchin
}

\section{To cite this version:}

Sandeep Kumar Chaluvadi, Fernando Ajejas, Pasquale Orgiani, Sylvain Lebargy, Albert Minj, et al.. Epitaxial strain and thickness dependent structural, electrical and magnetic properties of La0.67Sr0.33MnO3 films. Journal of Physics D: Applied Physics, 2020, 53, pp.375005. 10.1088/1361$6463 / \mathrm{ab} 8 \mathrm{e} 7 \mathrm{~b}$. hal-02568880

\section{HAL Id: hal-02568880 https://hal.science/hal-02568880}

Submitted on 10 May 2020

HAL is a multi-disciplinary open access archive for the deposit and dissemination of scientific research documents, whether they are published or not. The documents may come from teaching and research institutions in France or abroad, or from public or private research centers.
L'archive ouverte pluridisciplinaire HAL, est destinée au dépôt et à la diffusion de documents scientifiques de niveau recherche, publiés ou non, émanant des établissements d'enseignement et de recherche français ou étrangers, des laboratoires publics ou privés. 
ACCEPTED MANUSCRIPT

\section{Epitaxial strain and thickness dependent structural, electrical and magnetic properties of La $<$ sub $>0.67</$ sub $>$ Sr $<$ sub $>0.33</$ sub $>$ MnO $<$ sub $>3</$ sub $>$ films}

To cite this article before publication: Sandeep Kumar Chaluvadi et al 2020 J. Phys. D: Appl. Phys. in press https://doi.org/10.1088/1361$\underline{6463 / a b 8 e 7 b}$

\section{Manuscript version: Accepted Manuscript \\ Accepted Manuscript is "the version of the article accepted for publication including all changes made as a result of the peer review process, and which may also include the addition to the article by IOP Publishing of a header, an article ID, a cover sheet and/or an 'Accepted Manuscript' watermark, but excluding any other editing, typesetting or other changes made by IOP Publishing and/or its licensors"}

This Accepted Manuscript is @ 2020 IOP Publishing Ltd.

During the embargo period (the 12 month period from the publication of the Version of Record of this article), the Accepted Manuscript is fully protected by copyright and cannot be reused or reposted elsewhere.

As the Version of Record of this article is going to be / has been published on a subscription basis, this Accepted Manuscript is available for reuse under a CC BY-NC-ND 3.0 licence after the 12 month embargo period.

After the embargo period, everyone is permitted to use copy and redistribute this article for non-commercial purposes only, provided that they adhere to all the terms of the licence https://creativecommons.org/licences/by-nc-nd/3.0

Although reasonable endeavours have been taken to obtain all necessary permissions from third parties to include their copyrighted content within this article, their full citation and copyright line may not be present in this Accepted Manuscript version. Before using any content from this article, please refer to the Version of Record on IOPscience once published for full citation and copyright details, as permissions will likely be required. All third party content is fully copyright protected, unless specifically stated otherwise in the figure caption in the Version of Record.

View the article online for updates and enhancements. 


\title{
Epitaxial strain and thickness dependent structural, electrical and magnetic properties of $\mathrm{La}_{0.67} \mathrm{Sr}_{0.33} \mathrm{MnO}_{3}$ films
}

Sandeep Kumar Chaluvadi ${ }^{1,2}$, Fernando Ajejas ${ }^{3,4}$, Pasquale Orgiani ${ }^{2,5}$, Sylvain Lebargy ${ }^{1}$, Albert ${ }^{\text {Minj }}{ }^{1}$, Stephane Flament $^{1}$, Julio Camerero ${ }^{3,4}$, Paolo Perna ${ }^{3}$, and Laurence Méchin ${ }^{1}$

${ }^{1}$ Normandie Univ, UNICAEN, ENSICAEN, CNRS, GREYC, 14000 Caen, France

${ }^{2}$ CNR-IOM TASC National Laboratory, Area Science Park-Basovizza, 34149 Trieste, Italy

${ }^{3}$ IMDEA Nanociencia, Campus de Cantoblanco, 28049 Madrid, Spain

${ }^{4}$ Universidad Autónoma de Madrid, Campus de Cantoblanco, 28049 Madrid, Spain

${ }^{5}$ CNR-SPIN, UOS Salerno, 84084 Fisciano, SA, Italy

E-mail: laurence.mechin@ensicaen.fr

\begin{abstract}
The crystal structural quality and the strain induced by the substrate strictly impose the magnetic and transport properties of $\mathrm{La}_{0.67} \mathrm{Sr}_{0.33} \mathrm{MnO}_{3}$ (LSMO) films. In particular, the magnetic anisotropy (MA) of epitaxial LSMO can be finely tuned by varying its thickness and by choosing single crystal substrates with suitable lattice mismatch with the film. Here, we have deposited LSMO films with thicknesses in the 12-50 $\mathrm{nm}$ range by pulsed laser deposition on different single crystal substrates inducing either compressive or tensile in-plane strain on the manganites. The epitaxial quality of films was quantified by $\omega$-scans around (002) peak with full-width half-maximum (FWHM) values as low as $0.08^{\circ}$ for films on the nearly matched NGO (110) substrate to $1.4^{\circ}$ films on high mismatched MgO (001) substrate. As the epitaxial strain in thin-film increases, a significant reduction in metal-insulation transition (MIT) temperature $\left(\mathrm{T}_{\mathrm{p}}\right)$ was observed. The magnetic properties of the films probed by Kerr magnetometry show that the symmetry of the room temperature MA varies significantly as a function of both strain and thickness. Specifically, we observed pure uniaxial MA on NGO (110) and pure biaxial MA on STO buffered $\mathrm{MgO}$ (001), whereas a spin reorientation from uniaxial in-plane to out-of-plane on LSAT (001) and uniaxial to nearly isotropic in-plane on STO (001) substrate as the film thickness is increased. We provide an efficient tool to tune the MA according to the specific spintronic application targeted.
\end{abstract}

Keywords: manganites, thin films, strain, magnetic anisotropy, spintronics 


\section{Introduction}

In any kind of magnetic materials, having control of magnetic anisotropy (MA) is fundamental for the development of devices. In thin-film technology, MA can be controlled by film thickness, growth mechanisms, and strain-induced by the substrate. The latter is a widely used effective tool to alter the properties of correlated functional oxide perovskite thin films. Strain (compressive and tensile) in $\mathrm{La}_{0.67} \mathrm{Sr}_{0.33} \mathrm{MnO}_{3}$ (LSMO) directly affects the $\mathrm{BO}_{6}$ octahedra, Mn-O-Mn bond angle [1] which in turn alters MIT, Curie temperature $\left(\mathrm{T}_{\mathrm{c}}\right)$ [2], and MA symmetry. Depending on the application, different magnetic anisotropic systems are to be chosen. For example, the uniaxial (two-fold) magnetic anisotropic system is used in developing magnetic field sensors based on anisotropic magneto-resistive (AMR) property [3] and biaxial (four-fold) magnetic anisotropic system can be used in four state memory and logic devices $[4,5]$.

Bulk LSMO with rhombohedral structure shows magneto-crystalline anisotropy with easy magnetization axis aligned with the $\{111\}$ crystal direction [6]. Whereas, in thin-film form, lattice misfit strain between substrate and film changes the magnetic anisotropy symmetry landscape, which substantially differs from the bulk. The $3 \mathrm{~d}$ electron occupancy in ' $\mathrm{e}_{\mathrm{g}}$ ' orbital for the films under tensile (compressive) strain favors $\mathrm{x}^{2}-\mathrm{y}^{2}\left(3 \mathrm{z}^{2}-\mathrm{r}^{2}\right)$ which orients the easy axis magnetization in-plane (out-ofplane). Several studies reported on the substrate-induced effects in LSMO films grown on STO (001) [79], STO (110) [8,10,11], NGO (110) [12], LSAT (001) [13,14], buffered Si [15]. However, a systematic study of the MA symmetry as a function of both thickness and substrate-induced lattice misfit strain is still lacking.

Here, we report on the structural, morphological, electrical properties as determined by specific MA of epitaxial LSMO thin films grown by Pulsed Laser Deposition (PLD) technique. The film thicknesses were varied within the $12-50 \mathrm{~nm}$ range, whereas the strain was varied by film-substrate lattice mismatch. The substrates were wisely chosen so that the films experience a wide range of strain states i.e., large compressive strain, nearly matched and large tensile strain. Our study will act as a template providing the missing link for choosing the right substrate and thickness of the film for the development of magnetic anisotropic based devices.

\section{Experimental details}

Epitaxial LSMO thin films were grown on different lattice-mismatched single substrates that includes inplane compressive strain on $\mathrm{LaAlO}_{3}$ (LAO) (001, -1.36\%), nearly matched on $\mathrm{NdGaO}_{3}$ (NGO) (110, $0.31 \%$ ) and $\mathrm{La}_{0.3} \mathrm{Sr}_{0.7} \mathrm{Al}_{0.65} \mathrm{Ta}_{0.35} \mathrm{O}_{3}$ (LSAT) (001, -0.12\%); in-plane tensile strain on $\mathrm{SrTiO}_{3}(\mathrm{STO})$ (001, $+0.82 \%)$, STO buffered $\mathrm{MgO}(001,>0.82 \%)$ and $\mathrm{MgO}(001,+8.04 \%)$, respectively by PLD technique. Prior to deposition, the substrates were heated to $720^{\circ} \mathrm{C}$ in vacuum at the rate of $15^{\circ} \mathrm{C}$ per minute. During the deposition, the energy density of $\mathrm{KrF}$ excimer laser of $248 \mathrm{~nm}$ wavelength was maintained between 1.4-1.7 $\mathrm{J} \mathrm{cm}^{-2}$, the laser repetition rate at $3 \mathrm{~Hz}$, the target to substrate distance was kept at $5 \mathrm{~cm}$ and oxygen partial pressure $\left(\mathrm{P}_{\mathrm{O} 2}\right)$ was maintained at 0.35 mbar. After deposition, the substrates were cooled down to room temperature at the rate of $10^{\circ} \mathrm{C}$ per minute in 700 mbar oxygen environment. Three different LSMO film thicknesses such as 12,25 and $50 \mathrm{~nm}$ were deposited accordingly on all the abovementioned substrates. The thickness of the STO buffer layer grown on MgO substrates was $12 \mathrm{~nm}$. The LSMO film crystal structure and surface morphology were examined using PANalytical X'Pert four-circle X-Ray Diffraction (XRD), and Bruker Nanoscale 3 Atomic Force Microscopy (AFM). Temperaturedependent electrical and magnetic properties were measured using the standard four-probe technique and Superconducting Quantum Interference Device (SQUID), respectively. The in-plane magnetic anisotropy properties were measured using Vectorial Magneto Optical Kerr (V-MOKE) magnetometry [16]. 


\section{Results and discussion}

Figure 1(a) shows the scale bar presenting the lattice misfit strain between bulk LSMO and single-crystal substrates used in this study. The surface topography of $50 \mathrm{~nm}$ thick LSMO films on different substrates was studied by AFM operated in tapping mode in scan areas of $2 \mu \mathrm{m} \times 2 \mu \mathrm{m}$, as shown in Figure 1(b-g). Depending on the misfit strain, different growth modes have been observed. For instance, LSMO grown on LAO (001) (Figure 1(b)) and $\mathrm{MgO}(001)$ (Figure 1(f, g)) substrates experience large misfit strain and the AFM morphology can be interpreted in terms of Wolmer-weber "island" type growth mode with root mean square (RMS) roughness of $\sim 0.4 \mathrm{~nm}$. Whereas, the AFM morphology of LSMO grown on NGO (110), LSAT (001) and STO (001) (Figure 1(c-e)) substrates show Frank van der Merwe "layer-by-layer" growth mode with clear surface terraces and RMS roughness values are below 1-unit cell. As the misfit strain increases, for particular thickness, the roughness of the films increases as shown in Figure 1(h). This can be attributed to different film growth modes. As thickness increases from 12 to $50 \mathrm{~nm}$, the roughness of the films also increases which is consistent with the literature. In all the films, the roughness lies below $\sim 0.45 \mathrm{~nm}$, which can be considered as smooth surfaces.

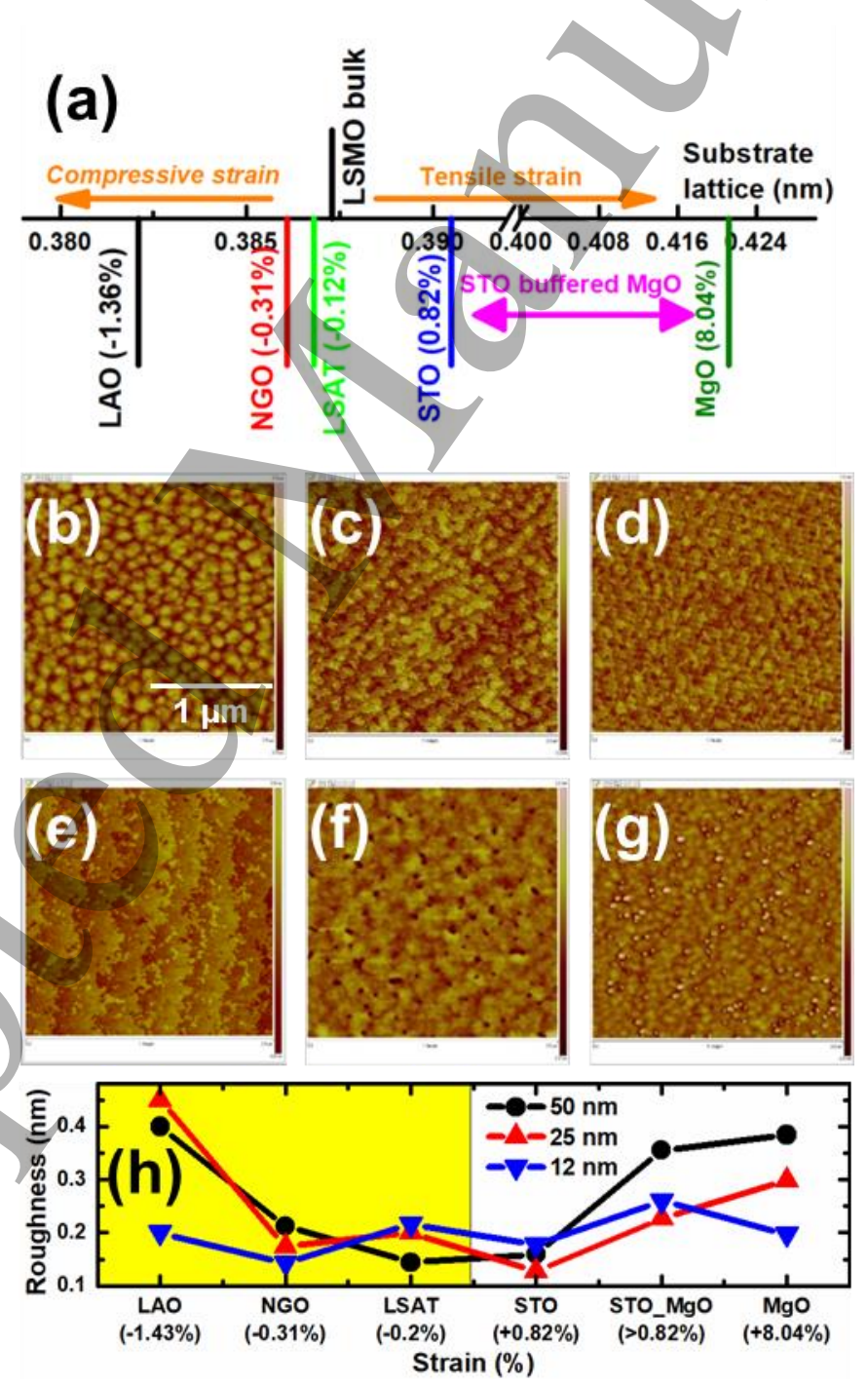

Figure 1. (Color online) (a) Scale-bar presenting the lattice parameters of different single crystal substrates used in this study along with the calculated lattice misfit strain by considering the pseudocubic bulk lattice parameter of LSMO $(0.389 \mathrm{~nm})$. (b-g) AFM topography images of $50 \mathrm{~nm}$ LSMO film grown 
on LAO, NGO, LSAT, STO, and STO buffered $\mathrm{MgO}$ and $\mathrm{MgO}$ substrates in the scan area of $2 \mu \mathrm{m} \times 2$ $\mu \mathrm{m}$, and (h) misfit strain-dependent RMS roughness of 50, 25 and $12 \mathrm{~nm}$ LSMO films.

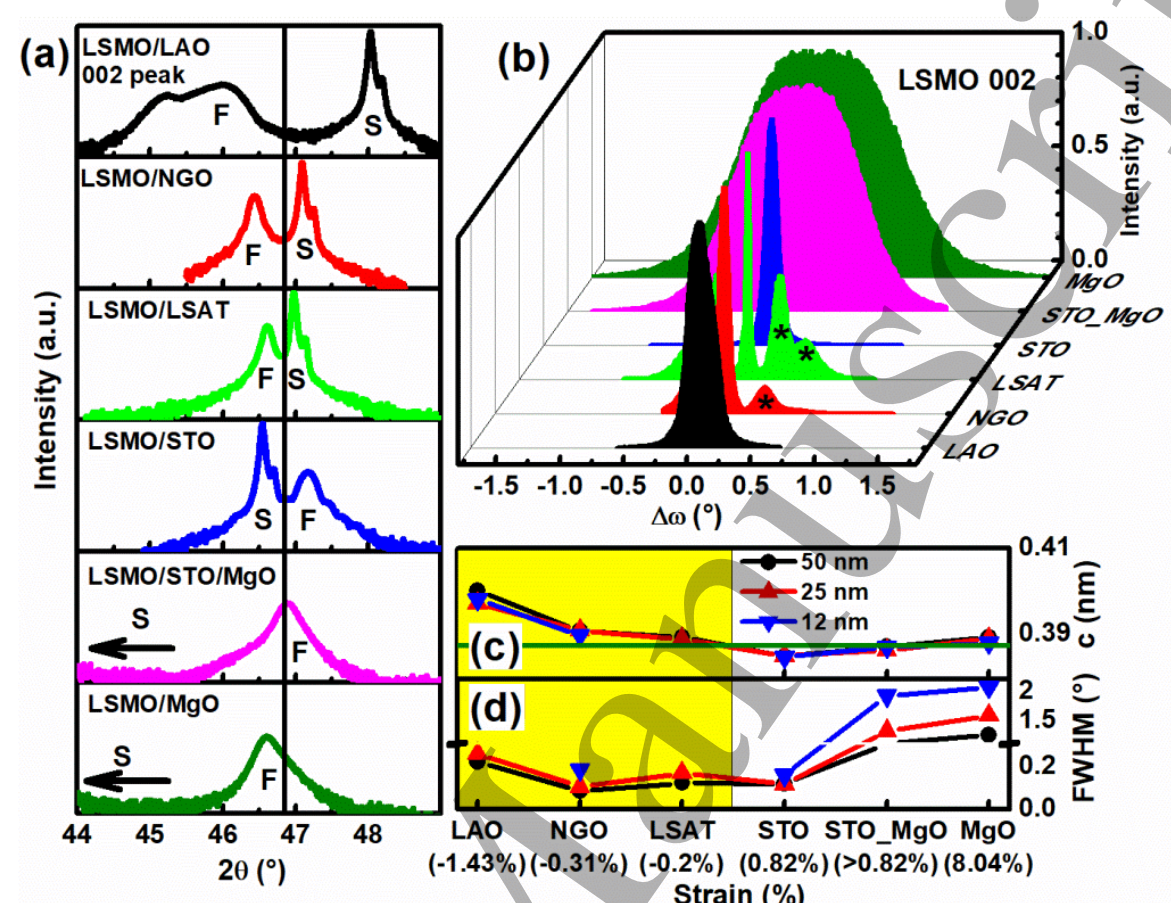

Figure 2. (Color online) (a) $\theta-2 \theta$ XRD measurements around the (002) crystallographic peak of $50 \mathrm{~nm}$ LSMO film deposited on different substrates. The letters ' $S$ ' and ' $F$ ' denotes substrate and film peaks, respectively. The solid vertical line indicates the peak position of bulk LSMO. (b) $\omega$-scans performed around the (002) peak of $50 \mathrm{~nm}$ LSMO film as a function of misfit strain. The notation ' $*$ ' in the NGO and LSAT denotes the satellite peaks. Thickness $(12-50 \mathrm{~nm})$ and misfit strain-dependent (c) out-of-plane lattice parameter calculated from $\theta-2 \theta$ scans, a solid horizontal line indicating the 'c' lattice parameter of pseudocubic bulk LSMO and (d) FWHM calculated from the (002) LSMO $\omega$-scans. Shaded and nonshaded regions represent compressive and tensile strain in the films, respectively.

The $\theta-2 \theta$ out-of-plane XRD measurements of $50 \mathrm{~nm}$ LSMO film in the scan area of $20^{\circ}-80^{\circ}$ are shown in the supplementary Figure S1 and confirm that all the films exhibit (OOl) epitaxial relationship without any spurious phases present in the films. Figure 2(a) shows the short-range $\theta-2 \theta$ around the (002) peaks of film and substrate as denoted by ' $F$ ' and ' $S$ ', whereas the solid vertical line indicates the pseudocubic peak position of bulk LSMO with lattice constant $a=0.3873 \mathrm{~nm}$. From the Bragg's diffraction law $(\mathrm{n} \lambda=2 \mathrm{~d} \sin \theta)$, it is known that the diffraction peak for a compressively (tensile) strained film shifts to lower (higher) angles from its bulk peak position and is attributed to the elongation (compression) of the out-of-plane lattice parameter ' $c$ ' of the unit cell. As expected, the 002 peak positions of LSMO film grown on LAO (001), NGO (110) and LSAT (001) substrates appeared on the left side of the bulk LSMO i.e., compressively strained and vice-versa for tensile strained. The out-ofplane lattice parameter ' $c$ ' for $50 \mathrm{~nm}$ film monotonically decreases as we move from compressive to tensile strained substrates such as $0.4 \mathrm{~nm}$ on LAO, $0.390 \mathrm{~nm}$ on NGO (110), $0.389 \mathrm{~nm}$ on LSAT and 0.385 on STO substrate. The film directly grown on $\mathrm{MgO}(001)$ appeared to be relaxed due to large interfacial strain. To overcome such relaxation, a thin $12 \mathrm{~nm}$ STO buffer layer has been introduced, which reduces interfacial strain and acts as a template to grow the good quality and epitaxially strained LSMO film. It is confirmed by $\theta-2 \theta$ XRD with the right shift in (002) LSMO peak from MgO (001) to STO 
buffered $\mathrm{MgO}$ substrate with the lattice constant 'c' $0.387 \mathrm{~nm}$. It is to be noted that due to the presence of multiple domains on LAO (001) substrate, a double peak has appeared on the $50 \mathrm{~nm}$ LSMO film. The measured out-of-plane lattice parameter ' $c$ ' of the film as a function of thickness and substrate-induced strain is shown in Figure 2(c) along with bulk LSMO value by a solid line for reference. As expected, as we move from the compressive to tensile strained film, the out-of-plane lattice parameter ' $c$ ' monotonically decreases from LAO to STO and reverses its trend on $\mathrm{MgO}$ substrate, which again indicates possible relaxation of the film.

In addition, more information can be extracted by analyzing the FWHM from the $\omega$-scans shown in Figure 2(b). The FWHM measured for the (002) LSMO peak of $50 \mathrm{~nm}$ on NGO (110), LSAT (001) and STO $(001)$ are $\sim 0.08^{\circ}, 0.12^{\circ}$, and $0.11^{\circ}$ respectively, indicating that the LSMO film planes are almost perfectly aligned with the substrate plane. As the lattice misfit strain increases, as expected, the peaks start to broaden and FWHM values increased from $0.3^{\circ}, 1^{\circ}$ to $1.3^{\circ}$ on LAO (001), STO buffered MgO (001) and $\mathrm{MgO}$ (001) substrates, respectively. Apart from the central peaks, it is interesting to observe satellite peaks (denoted as '*') on NGO (110) and LSAT (001) substrates. The observed satellite peaks are symmetrical to central peak and such observations are also found on similar systems [17-20] indicating a possible shear strain $[21,22]$ in the films. Thickness dependent LSMO (002) rocking curves on different substrates are shown in detail in the supplementary Figure S2. Figure 2(d) shows the evolution of the calculated FWHM as a function of strain and thickness. Films grown on the low mismatched substrate displays higher-quality growth with FWHM below $0.15^{\circ}$; whereas the FWHM increases to values as high as $2^{\circ}$ on large mismatched substrates ( $\mathrm{STO}$ buffered $\mathrm{MgO}$ and $\mathrm{MgO}$ ).

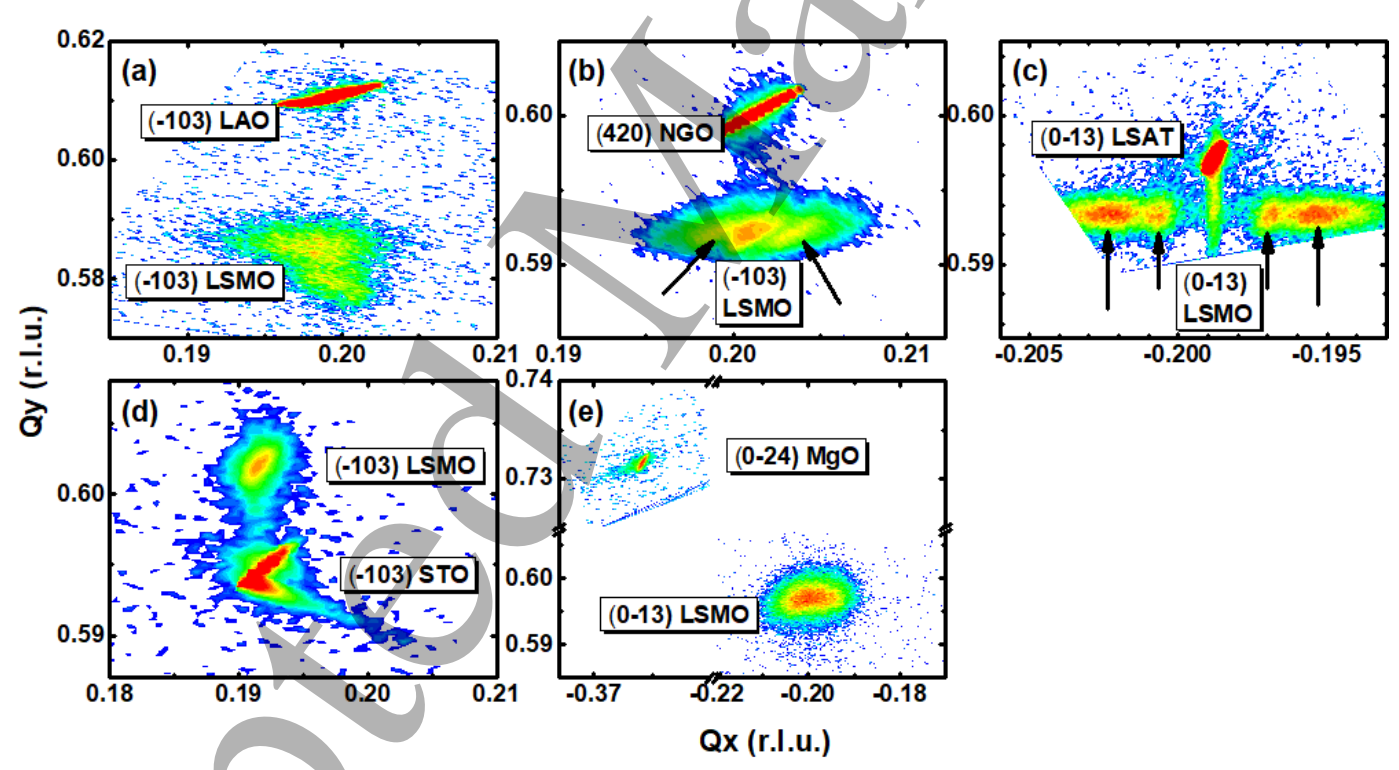

Figure 3. (Color online) Asymmetric reciprocal space maps around the $\{-103\}$ reflections of $50 \mathrm{~nm}$ LSMO film grown on (a) LAO (001), (b) NGO (110), (c) LSAT (001), (d) STO (001), and (e) MgO (001) respectively. The arrows in the (b and $\mathrm{c}$ ) indicate the satellite peaks.

Figure 3 shows the asymmetric reciprocal space maps (RSM) taken around $\{103\}$ reflections of $50 \mathrm{~nm}$ LSMO film grown on different substrates. It is seen that the $\mathrm{Q}_{x}$ position of the LSMO film is aligned vertically with LAO (001), NGO (110), LSAT (001) and STO (001) substrates (Figure 3(a-d)), thus confirming the pseudomorphic growth of LSMO. Therefore, the in-plane lattice parameters of the film are equivalent to the in-plane lattice parameters of the substrate. On the other hand, LSMO and MgO peaks do not align with the $\mathrm{Q}_{\mathrm{x}}$ position (Figure 3(e)), indicating the film tends to be relaxed with an average in-plane lattice parameter of $0.388 \mathrm{~nm}$ respectively. The satellite peaks observed in the $\omega$-scan (denoted by stars in Figure 2(b)) on NGO (110) and LSAT (001) also appear in the RSM experiments and 
are symmetric to the $\{103\}$ peak (arrows in Figure 3(b, c)). These satellite peaks (self-generated superlattices) could originate from another type of strain i.e., shear strain as observed in the previous reports [21].
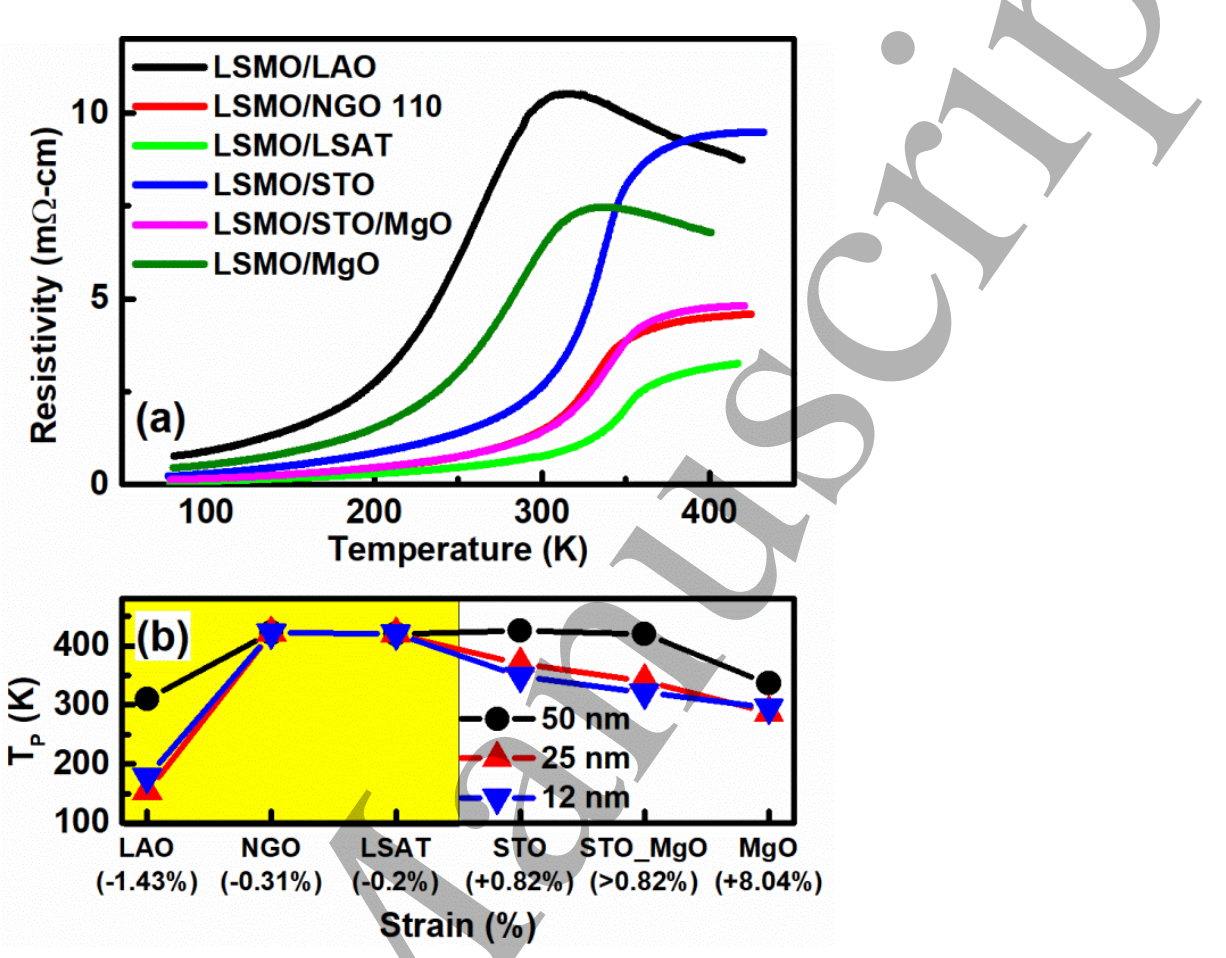

Figure 4. (Color online) (a) Temperature-dependent resistivity of $50 \mathrm{~nm}$ LSMO film deposited on various substrates. (b) Substrate misfit strain-dependent ' $T_{\mathrm{p}}$ ' of 50, 25 and $12 \mathrm{~nm}$ LSMO films. Shaded and nonshaded regions represent compressive and tensile strain in LSMO films.

Figure $4(\mathrm{a})$ shows the temperature-dependent $(77-420 \mathrm{~K})$ resistivity of $50 \mathrm{~nm}$ LSMO films deposited on different lattice-mismatched substrates. It is seen that the films that were grown on LSAT (001), NGO (110), STO (001) and STO buffered MgO (001) substrates display very low residual resistivity $(\rho<3 \mathrm{~m} \Omega-\mathrm{cm})$ at $300 \mathrm{~K}$ and $\mathrm{T}_{\mathrm{p}}$ well above $420 \mathrm{~K}$, indicating the films are of very high epitaxial quality. On the other hand, as the strain increases, the resistivity starts to increase and $\mathrm{T}_{\mathrm{p}}$ gradually decreases from $420 \mathrm{~K}$ on nearly matched substrates to $330 \mathrm{~K}$ on LAO (001) and $320 \mathrm{~K}$ on $\mathrm{MgO}(001)$ substrates, respectively. Thickness dependent resistivity curves on different substrates are shown in detail in the supplementary Figure S3. Figure 4(b) shows the thickness and misfit strain-dependent $T_{p}$, which indicates that the film under compressive strain (shaded region) exhibits higher ' $\mathrm{T}_{\mathrm{p}}$ ' compared to the films under tensile strain, which is quite consistent with the Millis [23] prediction. By comparing the same film thickness, the $T_{p}$ falls rapidly for the large misfit strained films. 


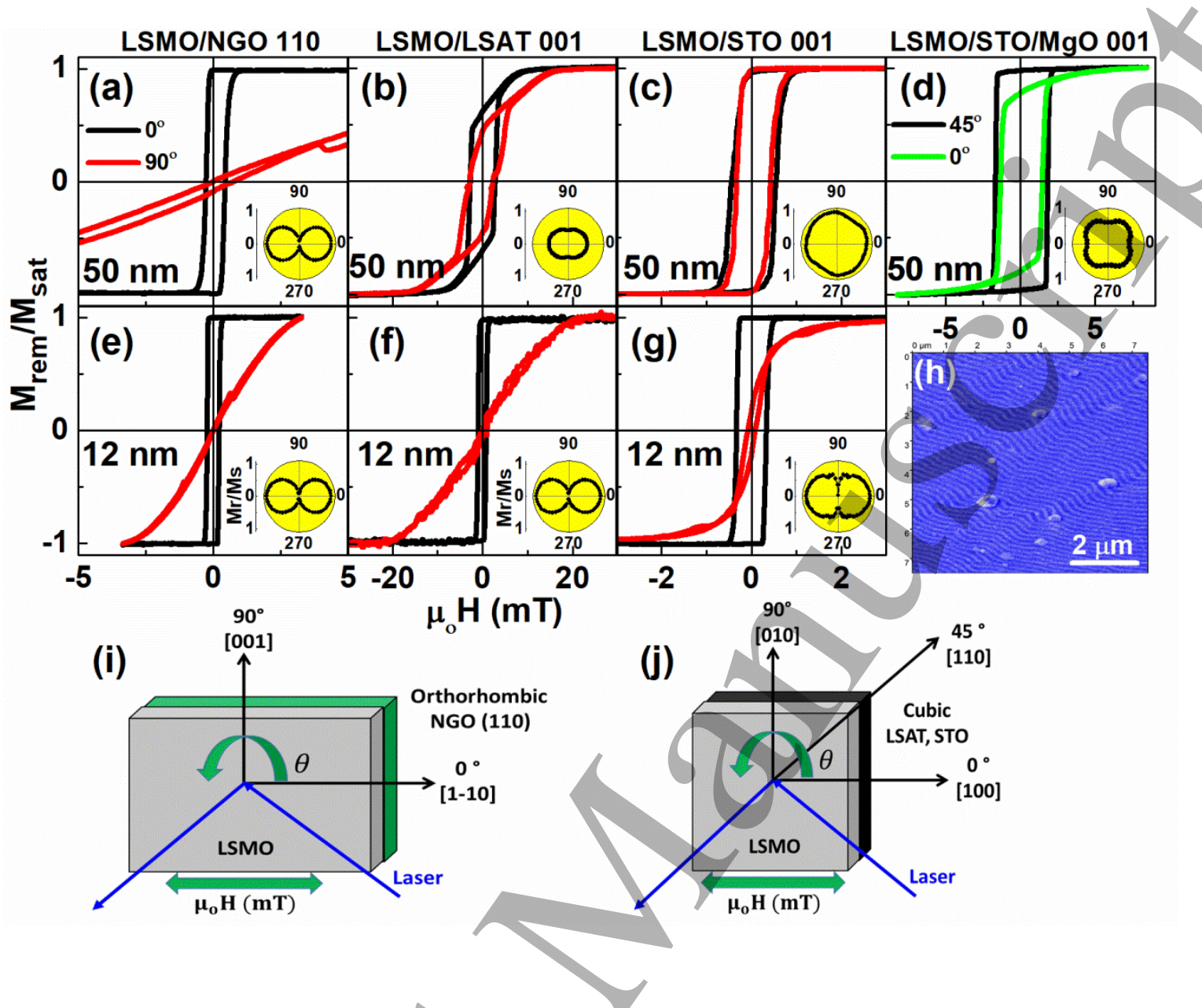

Figure 5. (Color online) Normalized in-plane magnetic hysteresis loops measured at their corresponding easy and hard axis of 50 and $12 \mathrm{~nm} \mathrm{LSMO} \mathrm{films} \mathrm{on} \mathrm{(a,} \mathrm{e)} \mathrm{NGO} \mathrm{(110)} \mathrm{at} \mathrm{[1-10]}\left(\theta=0^{\circ}\right)$ and [001] $\left(\theta=90^{\circ}\right)$, $(\mathrm{b}, \mathrm{f}) \operatorname{LSAT}(001)$ at $[100]\left(\theta=0^{\circ}\right)$ and $[010]\left(\theta=90^{\circ}\right),(\mathrm{c}, \mathrm{g}) \mathrm{STO}(001)$ at $[100]\left(\theta=0^{\circ}\right)$ and [010] $\left(\theta=90^{\circ}\right)$, and (d) $50 \mathrm{~nm}$ LSMO film on STO buffered $\mathrm{MgO}(001)$ at [110] $\left(\theta=45^{\circ}\right)$ and [100] $\left(\theta=0^{\circ}\right)$, respectively. Inset in (a-g) shows the angular dependent remanence polar plots of the respective films. (h) Magnetic force microscopy (MFM) image of $50 \mathrm{~nm}$ LSMO film on LSAT (001) substrate in the scan area of 7.5 $\mu \mathrm{m} \times 7.5 \mu \mathrm{m}$. (i, j) Schematic representation of the longitudinal MOKE configuration on orthorhombic and cubic substrates along with their crystallographic directions.

Figure 5(a-g) shows representative in-plane magnetic hysteresis loops acquired at the characteristic easy and hard axis of 12 and $50 \mathrm{~nm}$ LSMO films. The insets of Figure 5(a-g) show the corresponding angular-dependent remanence polar plots. When coherently grown onto an orthorhombic substrate such as NGO (110), the in-plane lattice parameters of LSMO matches with the in-plane lattice of NGO (110) substrate, inducing anisotropic in-plane strain due to dissimilarities in lattice constants along [1-10] and [001] in-plane directions [12]. Due to this anisotropic in-plane strain, the easy axis magnetization direction preferably aligns with the direction of higher stress [1-10] and the hard axis along the [001] direction. As the thickness increases, the strength of the uniaxial anisotropy i.e., anisotropy field $\mu_{0} \mathrm{H}_{k}$ also increases from 3.4 to $8.4 \mathrm{mT}$ (Figure 5(a, e)). Therefore, irrespective of the thickness range $(12-50 \mathrm{~nm})$, the film exhibits strong uniaxial anisotropy (two-fold symmetry) and is attributed to the stress anisotropy or magneto-elastic effects. 
LSMO grown onto a cubic substrate such as LSAT (001), the film is compressively strained. At low thickness $(12 \mathrm{~nm})$, the MA of the film exhibits pure uniaxial anisotropy i.e., remanence at the hard axis $\left([010]\right.$ or $\left.\theta=90^{\circ}\right)$ is zero (Figure $5(\mathrm{f})$ ). As the substrates are cubic, the in-plane strain is isotropic. Therefore, we could rule out the possible contribution from magneto-elastic effects as discussed on the orthorhombic NGO (110) substrate. This dominant uniaxial anisotropy contribution could be attributed due to interface effects, strain, octahedral rotations [24] and substrate mis-cut angles [9] etc. As the thickness increases, the volume of the film increases and the MA contributions due to interfacial and substrate-induced effects are overcome. The hysteresis loops of a $50 \mathrm{~nm}$ film on LSAT substrate measured at $0^{\circ}[100]$ and $90^{\circ}$ [010] (Figure 5(b)) do not display either a perfect square or linear loop indicating that the anisotropy is weak. It must be noted that the remanence present at an easy axis $\left(0^{\circ}\right.$ [100]) is $40 \%$ less than the saturation magnetization. This slope indicates that, along with in-plane magnetization contribution, there could also be an additional out-of-plane (OOP) component. To confirm such an OOP component, magnetic force microscopy (MFM) imaging is performed on $50 \mathrm{~nm}$ LSMO film in the scan area of $7.5 \mu \mathrm{m} \times 7.5 \mu \mathrm{m}$ as shown in Figure 5(h). As the MFM tip is sensitive to OOP magnetization component, MFM pattern observed with well-ordered periodic stripe patterns confirms the presence of OOP magnetic component [13]. Therefore, together with in-plane MOKE hysteresis loops Figure 5(b) and OOP MFM pattern Figure 5(h) confirms that the magnetization in $50 \mathrm{~nm}$ LSMO film constitutes both in-plane and OOP magnetization leading to canted anisotropy. In short, as the thickness of the LSMO film increases on LSAT (001) substrate, the compressive strain in the film overcomes interfacial effects and the OOP magnetization component tends to increase.

LSMO grown on cubic STO (001) substrate, the LSMO unit cell experiences equal tensile strain along with two in-plane directions i.e., [100] and [010] and the magnetization for the tensile strained films always lie in the plane of the film. At low thickness, i.e., $12 \mathrm{~nm}$, the easy and hard axis correspond to [100] $\left(\theta=0^{\circ}\right)$ and [010] $\left(\theta=90^{\circ}\right)$ direction (Figure 5(g)) with two-fold symmetry due to steps and miscut angles originated from the STO substrate. At higher thickness i.e., $50 \mathrm{~nm}$ (Figure 5(c)), the MA usually shows a competition between bulk (biaxial) [25] and weak shape or strain (uniaxial) magnetic anisotropy [26].

In the case of $50 \mathrm{~nm}$ LSMO film grown on STO buffered MgO (001) (Figure 5(d)), the easy and hard axes are present at $45^{\circ}$ (or [110]) and hard axis at $0^{\circ}$ (or [100]), respectively. The inset of Figure 5(d) shows four-fold symmetry (magnetocrystalline) with easy axis magnetization aligned with [110] and its equivalent pseudocubic axes as explained in detail in our previous study [27]. Table 1 gives in detail the summary of substrates, thickness, and anisotropy field, MA and its possible origins. It also gives some of the applications that can be used based on the MA symmetry.

Table 1: Thickness and substrate-dependent in-plane magnetic anisotropy, dominant effects that contributes to magnetc anistropy, anisotropic field and applications are tabulated.

\begin{tabular}{|c|c|c|c|c|c|}
\hline Substrates & $\begin{array}{l}\text { LSMO } \\
\text { thickness }\end{array}$ & $\begin{array}{l}\text { In-plane magnetic } \\
\text { anisotropy }\end{array}$ & Dominant effect & $\begin{array}{l}\boldsymbol{\mu}_{\mathbf{0}} \mathbf{H}_{\mathbf{k}} \\
(\mathbf{m T})\end{array}$ & Applications \\
\hline NGO (110) & $12-50 \mathrm{~nm}$ & Uniaxial & Magneto-elastic & $3.4-8.4$ & AMR sensors \\
\hline $\begin{array}{c}\text { LSAT } \\
(001)\end{array}$ & $\frac{50 \mathrm{~nm}}{12 \mathrm{~nm}}$ & $\frac{\text { Uniaxial + OOP }}{\text { Uniaxial }}$ & $\begin{array}{l}\text { Compressive strain, } \\
\text { interface effects }\end{array}$ & $\begin{array}{l}17 \\
20\end{array}$ & AMR sensors \\
\hline & $50 \mathrm{~nm}$ & $\begin{array}{l}\text { Biaxial (almost } \\
\text { isotropic) }\end{array}$ & $\begin{array}{l}\text { Magneto-crystalline + } \\
\text { step-induced }\end{array}$ & 3.3 & $\begin{array}{c}\text { User choice by } \\
\text { inducing shape } \\
\text { effects }\end{array}$ \\
\hline & $12 \mathrm{~nm}$ & Uniaxial & $\begin{array}{l}\text { Steps, miscut angle of } \\
\text { STO }\end{array}$ & 2 & AMR sensors \\
\hline $\begin{array}{c}\text { STO/MgO } \\
(001)\end{array}$ & $50 \mathrm{~nm}$ & Pure Biaxial & Magneto-crystalline & 5.47 & $\begin{array}{c}\text { Four-state } \\
\text { memory devices }\end{array}$ \\
\hline
\end{tabular}




\section{Conclusion}

We discussed structural, morphological, electrical transport and in-plane magnetic anisotropy properties of LSMO thin films as a function of film thickness and lattice misfit strain imposed by the underlying substrates. Films grown on low misfit strained substrates such as NGO (110), LSAT (001) and STO (001) are fully strained and displayed very low FWHM $\left(<0.2^{\circ}\right)$ and display high $T_{p}(>420 \mathrm{~K})$ and RMS roughness lies below 1-unit cell, indicating very high-quality of the films. The MA of films grown on orthorhombic NGO (unequal in-plane compressive strain) always displays uniaxial anisotropy irrespective of the thickness, while on cubic LSAT (equal in-plane compressive strain) substrates, the easy magnetization direction changes from in-plane to out-of-plane with an increase in thickness. Under tensile strain substrate as cubic STO (equal in-plane tensile strain), $12 \mathrm{~nm}$ LSMO films present uniaxial in-plane MA owing to substrate-induced effects as steps, miscut angles etc. As the thickness of the films increases, substrate effects overcome and display weak biaxial in-plane anisotropy owing to magnetocrystalline of LSMO. Finally, by adding a thin buffer layer of STO onto MgO (001) substrate, LSMO films exhibit pure biaxial in-plane MA corresponds to the magneto-crystallinity nature of bulk LSMO. The understanding of the substrate and thickness dependent MA in LSMO, which directly affects the magnetoresistance, is essential for the development of manganites-based magnetic devices.

See Supplementary information for the detailed structural, electrical transport properties of LSMO films on various substrates and thicknesses.

\section{References}

[1] Vailionis A, Boschker H, Siemons W, Houwman E P, Blank D H A, Rijnders G and Koster G 2011 Misfit strain accommodation in epitaxial ABO3 perovskites: Lattice rotations and lattice modulations Phys. Rev. B 8364101

[2] Adamo C, Ke X, Wang H Q, Xin H L, Heeg T, Hawley M E, Zander W, Schubert J, Schiffer P, Muller D a., Maritato L and Schlom D G 2009 Effect of biaxial strain on the electrical and magnetic properties of (001) La0.7Sr0.3MnO3 thin films Appl. Phys. Lett. 95112504

[3] Perna P, Maccariello D, Ajejas F, Guerrero R, Méchin L, Flament S, Santamaria J, Miranda R and Camarero J 2017 Engineering Large Anisotropic Magnetoresistance in La 0.7 Sr 0.3 MnO 3 Films at Room Temperature Adv. Funct. Mater. 271700664

[4] Salehi-Fashami M and D'Souza N 2017 Exploring performance, coherence, and clocking of magnetization in multiferroic four-state nanomagnets J. Magn. Magn. Mater. 438 76-84

[5] M.V.Pitke 1971 Biaxial anisotropy for memory applications Czech J Phys B 21 467-9

[6] Konoto M, Kohashi T, Koike K, Arima T, Kaneko Y, Tomioka Y and Tokura Y 2004 Magnetic domain structure of a $\mathrm{La} 0.7 \mathrm{Sr} 0.3 \mathrm{MnO} 3$ (001) surface observed by a spin-polarized scanning electron microscope Appl. Phys. Lett. 84 2361-3

[7] Mathews M, Postma F M, Lodder J C, Jansen R, Rijnders G and Blank D H A 2005 Step-induced uniaxial magnetic anisotropy of La0:67Sr0:33MnO3 thin films Appl. Phys. Lett. 87242507

[8] Perna P, Rodrigo C, Jiménez E, Teran F J, Mikuszeit N, Méchin L, Camarero J and Miranda R 2011 Tailoring magnetic anisotropy in epitaxial half metallic La0.7SrO.3MnO3 thin films J. Appl. Phys. 11013919

[9] Chaluvadi S K, Perna P, Ajejas F, Camarero J, Pautrat A, Flament S and Méchin L 2017 Thickness and angular dependent magnetic anisotropy of La $0.67 \mathrm{Sr} 0.33 \mathrm{MnO} 3$ thin films by Vectorial Magneto Optical Kerr Magnetometry J. Phys. Conf. Ser. 90312021

[10] Boschker H, Kautz J, Houwman E P, Koster G, Blank D H a. and Rijnders G 2010 Magnetic anisotropy and magnetization reversal of $\mathrm{La} 0.67 \mathrm{Sr} 0.33 \mathrm{MnO} 3$ thin films on SrTiO3(110) J. Appl. Phys 108103906

[11] Wang Z-H, Kronmüller H, Lebedev O, Gross G, Razavi F, Habermeier H and Shen B 2002 Phase transition and magnetic anisotropy of (La,Sr)MnO3 thin films Phys. Rev. B 6554411

[12] Mathews M, Jansen R, Rijnders G, Lodder J C and Blank D H A 2009 Magnetic oxide nanowires with strain-controlled uniaxial magnetic anisotropy direction Phys. Rev. B $\mathbf{8 0} 64408$ 
[13] Méchin L, Simon C and Chakalov R A 2008 Variable temperature magnetic force microscopy Int. J. Nanotechnol. 5 818-26

[14] Boschker H, Mathews M, Brinks P, Houwman E, Vailionis A, Koster G, Blank D H A and Rijnders G 2011 Uniaxial contribution to the magnetic anisotropy of $\mathrm{La0} .67 \mathrm{Sr} 0.33 \mathrm{MnO} 3$ thin films induced by orthorhombic crystal structure J. Magn. Magn. Mater. 323 2632-8

[15] Perna P, Méchin L, Chauvat M P, Ruterana P, Simon C and Scotti di Uccio U 2009 High Curie temperature for $\mathrm{La}(0.7) \mathrm{Sr}(0.3) \mathrm{MnO}(3)$ thin films deposited on $\mathrm{CeO}(2) /$ YSZ-based buffered silicon substrates. J. Phys. Condens. Matter 21306005

[16] Jiménez E, Mikuszeit N, Cuñado J L F, Perna P, Pedrosa J, Maccariello D, Rodrigo C, Niño M A, Bollero A, Camarero J and Miranda R 2014 Vectorial Kerr magnetometer for simultaneous and quantitative measurements of the in-plane magnetization components Rev. Sci. Instrum. 8553904

[17] Santiso J, Balcells L, Konstantinovic Z, Roqueta J, Ferrer P, Pomar A, Martínez B and Sandiumenge F 2013 Thickness evolution of the twin structure and shear strain in LSMO films CrystEngComm 153908

[18] Sandiumenge F, Santiso J, Balcells L, Konstantinovic Z, Roqueta J, Pomar A, Espinós J P and Martínez B 2013 Competing misfit relaxation mechanisms in epitaxial correlated oxides Phys. Rev. Lett. 110 1-5

[19] Jin S, Gao G, Wu W and Zhou X 2007 Effect of angular-distortion-induced strain on structural and transport properties of epitaxial La0.7Sr0.3MnO3 thin films J. Phys. D. Appl. Phys. 40 305-9

[20] Lebedevy O I, Amelinckx S and Razavi F 2001 Periodic microtwinning as a possible mechanism for the accommodation of the epitaxial film-substrate mismatch in the La1-xSrxMnO3/SrTiO 3 system Philos. Mag. A 81 797-824

[21] Zhou T F, Li G, Li X G, Jin S W and Wu W B 2007 Self-generated in-plane superlattice in relaxed epitaxial La 0.67Sr0.33MnO3 films Appl. Phys. Lett. 90 0-3

[22] Jin S W, Gao G Y, Huang Z, Yin Z Z, Zheng X and Wu W 2008 Shear-strain-induced low symmetry phase and domain ordering in epitaxial La0.7Sr0.3MnO3 thin films Appl. Phys. Lett. 92 $1-4$

[23] Millis A J, Darling T and Migliori A 1998 Quantifying strain dependence in "colossal" magnetoresistance manganites J. Appl. Phys. 83 1588-91

[24] Liao Z, Huijben M, Zhong Z, Gauquelin N, Macke S, Green R J, Van Aert S, Verbeeck J, Van Tendeloo G, Held K, Sawatzky G A, Koster G, Rijnders G, Z. Zhong, Gauquelin N, Macke S, Green R J, Aert S van, Verbeeck J, Tendeloo G Van, Held K, Sawatzky G A, Koster G and Rijnders G 2016 Controlled lateral anisotropy in correlated manganite heterostructures by interface-engineered oxygen octahedral coupling. Nat. Mater. 15 425-31

[25] Suzuki Y, Hwang H Y, Cheong S-W and van Dover R B 1997 The role of strain in magnetic anisotropy of manganite thin films Appl. Phys. Lett. 71 140-2

[26] Reeve R M, Mix C, König M, Foerster M, Jakob G and Kläui M 2013 Magnetic domain structure of $\mathrm{La} 0.7 \mathrm{Sr} 0.3 \mathrm{MnO} 3$ thin-films probed at variable temperature with scanning electron microscopy with polarization analysis Appl. Phys. Lett. 102122407

[27] Chaluvadi S K, Ajejas F, Orgiani P, Rousseau O, Vinai G, Petrov A Y, Torelli P, Pautrat A, Camarero J, Perna P and Mechin L 2018 Room temperature biaxial magnetic anisotropy in

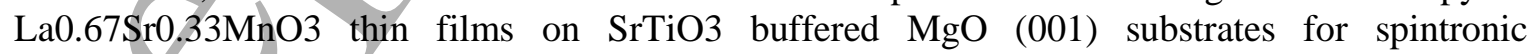
applications Appl. Phys. Lett. 11352403 